\title{
Throne of Glass, Celaena Sardothien et la violence au féminin
}

\author{
Fanie DEMEULE, Université du Québec à Montréal
}

Il suffit d'entrer dans la section adolescente d'une librairie pour constater que le royaume de la young adult fiction, c'est-à-dire de la littérature pour jeune adulte, est devenu le territoire de prédilection d'un certain type d'héroïne guerrière qui se bat physiquement. En effet, à l'image de Katniss Everdeen de la franchise The Hunger Games, un bataillon d'œuvres de fiction destinées à un lectorat jeunesse met désormais en vedette des personnages de jeunes filles armées. Cet article vise à montrer que si la jeune guerrière connaît une visibilité et une popularité grandissante, les représentations de sa violence et son agressivité relèvent d'une difficulté persistante à concevoir une cohabitation entre le genre féminin et le comportement violent. Nous étudierons comment la violence, potentiellement perturbante pour le lectorat, de Celaena Sardothien est négociée dans la série Throne of Glass. Pour ce faire, nous regarderons la constitution identitaire du personnage, son iconographie, pour finalement examiner la polémique entourant sa réception au sein du lectorat.

\section{Au royaume de la fantasy jeunesse}

La série romanesque Throne of Glass, de l'auteure américaine Sarah J. Mass, est jusqu'à présent composée de cinq volets, dont un recueil de nouvelles. Campée dans un univers de fantasy, cette série, explicitement destinée à des adolescentes, suit les pas de Celaena Sardothien, héroïne âgée de seize ans, à travers sa quête de liberté. Alors que nous apprenons à la fin du deuxième tome que Celaena s'appelle véritablement Aelin Ashryver Galathynius, dans le cadre de cet article, nous l'appellerons uniquement Celaena. En effet, Celaena Sardothien n'est que le premier d'une suite de pseudonymes qu'elle s'attribue ou qui lui sont donnés au fil de ses transformations sociales, qui traduisent sa malléabilité identitaire. La jolie jeune femme blonde est candidate pour le titre d'assassin royal lors d'une compétition opposant des combattants

exclusivement masculins, concours qu'elle remporte haut la main et acquière le titre de Championne du Roi d'Adarlan. Détenant l'exclusivité féminine à la cour militaire, elle n'est toutefois pas la seule héroïne guerrière de l'univers de Throne of Glass, constellé de combattantes. 
En tant que princesse exilée du Royaume de Terrasen, son objectif principal est de regagner ses terres afin de les faire renaitre de leurs cendres, leur ruine ayant été causée par le souverain dont elle est à la solde. Outre l'apprentissage des arts de la guerre et des stratégies politiques, le parcours de Celaena, à l'image de celui de la plupart des héroïnes guerrières en fiction pour jeune adulte, illustre sur un mode fantastique le passage complexe de l'enfance vers le monde adulte. Celaena doit ainsi apprendre à maîtriser ses dons particuliers de magie du feu, tout en tentant de démêler l'étrange dimension des sentiments amoureux et de la sexualité. Elle est une «jeune fille en feu », et non en «fleur », dans le sens où l'apprentissage du contrôle de son feu, qui à la fois la forge et la détruit, devient gage métaphorique de sa maturation.

\section{Vision dualiste}

Maas, l'auteure de la série, explique avoir voulu créer une héroïne guerrière forte à travers laquelle ses jeunes lectrices reconnaîtraient un modèle d'agentivité : «I'd love for some young woman to read [Throne of Glass] and feel empowered », confirme-t-elle lors d'une interview (Gallucci 1). D'ailleurs, le mantra que se récite l'hérö̈ne pour se donner du courage lors des épreuves à surmonter en est un de confiance en soi : «My name is Celaena Sardothien and I will not be afraid» (20). En créant sa protagoniste, l'auteure explique s'être inspirée de divers personnages féminins et masculins issus de la culture populaire en les amalgamant librement : elle allie, entre autres, la force du chevalier à la coquetterie de la princesse. Si l'on se fie à la manière dont Maas conçoit son héroïne, Celaena se concevrait comme un personnage de sexe féminin actif qui affranchit les limites des genres. Afin de pouvoir considérer s'il y a construction liminale chez Celaena dans Throne of Glass, il est important de clarifier la nature de la conception idéologique dans laquelle nous nous positionnons.

Selon Diana Fuss, l' «essentialisme» repose sur une compréhension des concepts identitaires comme immuables et hors de portée de tout discours historique ou social (5). La vision essentialiste est par définition stéréotypée étant donné qu'elle s'inscrit dans une pensée binaire rattachant le genre au sexe de l'individu. Pour cet article, nous abonderons dans la logique de la culture de masse et des clichés essentialistes qui y sont reconduits. Ce choix relève d'une volonté de cohérence, car nous voulons comprendre comment l'imaginaire populaire construit le genre à partir d'une forme de stéréotypie des genres en la culture populaire et comment nous pouvons en observer l'influence sur l'audience dans ses façons de reconstruire 
l'identité. Nous traiterons donc d'une certaine tradition de la féminité et de la masculinité qui nous vient des imaginaires collectifs du fait que l'œuvre de Maas s'inscrit dans la culture populaire ${ }^{1}$. Pour cela, il nous faut d'abord déterminer de quels canons découle cette conception binaire des genres dans les productions grand public.

\section{Féminité, violence et liminalité}

Si l'on rejoint l'idée d'Hélène Cixous selon laquelle la féminité traditionnelle s'inscrit dans un canon religieux associé au culte de la Vierge, la féminité exemplaire serait silencieuse, statique et discrète, douce et protectrice, et présenterait un «maintien divin » loin de toute forme d'action et de recherche de pouvoir (246), contenance que l'on peut convertir en termes de nonviolence. Suivant ce schéma, la masculinité serait le contraire de la féminité, soit la prise de parole, l'action et l'exubérance. Cette conception binaire du genre s'appuie sur une idéologie dualiste qui régit une distinction nette entre les sexes, dichotomie que vient perturber la représentation de ce que Kathleen Rowe considère la «unruly woman ». Rowe explique que la rébellion de la femme ingouvernable contre son statut social non seulement renverse les distinctions hiérarchiques, c'est-à-dire celles entre homme et femme, mais les transcende. Ce faisant, le personnage ne devient pas un homme, mais ne demeure pas non plus une femme.

Tentons de lire le personnage de Celaena en parallèle avec cet archétype féminin de la unruly woman qui semble corroborer le projet littéraire de Maas. Selon Rowe, la femme ingouvernable est une figure qui perturbe l'image de la féminité stéréotypée en déroutant les attentes à l'égard de la féminité, entre autres en s'écartant de la passivité qu'elle se devrait d'adopter. Jusqu'à présent, le personnage de Celaena s'intègre à la conception de la unruly woman : la jeune héroïne possède une grande force physique, parle fort et avec rudesse, tout en cultivant un humour impitoyable. Elle fait aussi preuve d'une arrogance, ainsi qu'en témoigne sa réplique la plus célèbre : «My name is Celaena Sardothien, but it makes no difference if my name's Celaena or Lilian or Bitch, because I'd still beat you, no matter what you call me » (80). Son attitude irrévérencieuse correspond à l'archétype de femme en tant que sujet postulé par Rowe.

L'aspect subversif de la femme ingouvernable réside en sa cohabitation libre d'éléments issus des stéréotypes féminins et masculins, s'affranchissant ainsi de l'une des distinctions sacrées, soit celle entre les genres sexuels. À ce propos, la liminalité de genre est généralement 
problématique pour la femme guerrière, car celle-ci entretient d'emblée un rapport conflictuel avec la violence, élément masculin. La guerrière négocie la représentation de sa violence en vue d'un compromis permettant l'acceptation de son personnage chez le lectorat. Cette négociation explique le morcellement genré en tant que procédé évitant la contamination de la jeune fille avec la violence. En effet, si elle conserve l'un et l'autre, c'est-à-dire la féminité et la violence, l'usage de cette dernière s'accomplit suite à un passage vers une performance du genre masculin qui lui permet de rendre légitime sa violence. Pour reprendre la terminologie de Sherrie Inness, l'héroïne « s'endurcit » physiquement et psychologiquement avant de se battre. Non seulement elle revêt l'armure, mais sa personnalité se rigidifie et devient moins avenante. Lors de combat, la personnalité empathique de Celaena se transforme en une attitude irrévérencieuse et impitoyable.

Sur le plan de la représentation, chez Celaena comme chez la plupart des guerrières en young adult fiction, l'accoutrement tend à formater la physionomie dans une esthétique soit hyperféminine ou androgyne. L'héroïne possède deux garde-robes bien distinctes, l'une étant constituée de robes, jupes et autres vêtements délicats aux ajustements flatteurs, l'autre garnie de tenues de combat aux matières rudes et aux coupes garçonnes. Alors que le héros masculin traverse le récit ou l'écran toujours revêtu d'une sempiternelle tenue de combat, la guerrière se démène entre deux costumes, comme dépossédée d'un territoire vestimentaire neutre. Dans Throne of Glass, du côté des évènements mondains à la cour ou lors de missions spéciales dans le milieu aristocratique, Celaena doit projeter l'image d'une jeune femme ingénue et innocente en revêtant des robes et un maquillage travaillé, tandis que du côté des assassins et des guerriers, c'est une apparence plus masculine et ténébreuse qu'elle s'efforcer d'adopter. La narratrice réitère d'ailleurs explicitement vouer un véritable culte à son apparence, et c'est avec une précision obsessionnelle que celle-ci décrit toutes les séances de maquillage et d'habillage rencontrées au cours de ses aventures : "After nearly two hours of pampering - trimming her hair, shaping her nails, and scraping away the callouses on her feet and hands - Celaena grinned at the mirror in the dressing room. [...] She looked spectacular. Utterly and completely spectacular » (45).

Les élaborations de ses mascarades nous sont ainsi longuement révélées, témoignant des efforts requis pour la mise en place de son image genrée et de la facticité de celle-ci. Par ailleurs, les deux codes vestimentaires de Celaena Sardothien sont clairement suggérés de 
manière dichotomique à travers le paratexte. Par exemple, sur les illustrations de première et de quatrième de couverture de chaque tome, la page de couverture la présente en tenue de combat tandis que le verso lui fait arborer la robe. Il est intéressant de noter que sur les illustrations, malgré la polarisation des iconographies, l'héroïne pose en adoptant une gestuelle et une arme similaires sur la première et la quatrième de couverture. Ces illustrations ont-elles pour but de provoquer la curiosité en raison de leur caractère incongru, de vendre un certain type de femme féminine et armé? Quoi qu'il en soit, s’il est représenté sur les couvertures, ce phénomène de juxtaposition n'advient toutefois pas dans le récit, car l'assassine ne se battra jamais en tenue féminine.

On peut comprendre l'adoption d'une fragmentation du personnage comme une forme de conciliation visant la préservation de son capital féminin non violent. Ainsi, la renonciation de la féminité observable chez la plupart des guerrières est moins un rejet qu'un sacrifice permettant de départager la féminité de la violence, dans le sens où sacrifier revient à séparer le profane du sacré (Dufourmantelle 2). Pour faire résonner Giorgio Agamben avec Mary Douglas, lesquels traitent de contrôle de la contamination idéologie en termes de séparation entre les éléments profanes et sacrés, on peut sacraliser un objet ou un individu en le retirant à l'usage courant. Pour Douglas : «The danger is controlled by ritual which precisely separates [the object] from his old status, segregates him for a time and then publicly declares his entry to his new status » (120). L'image de la féminité sacrée non violente contrebalance la peur d'une femme instable, violente et profane. La féminité et la violence se voient ainsi dissociées l'une de l'autre. Cette idée de préservation de la féminité dans un espace sacré rejoint la pensée d'Agamben pour qui consacrer quoi? « désign[e] la sortie des choses de la sphère du droit humain »(55). De cette façon, la violence proférée par la guerrière n'est pas complètement profanatrice, et elle laisse intacte la féminité.

Yvonne Tasker remarque qu'un récit centré sur une héroïne tend à expliquer le comportement agressif de celle-ci. De manière presque systématique, lorsque la violence de la guerrière est injustifiée et monstrueuse, le récit dépeint cette dernière comme fautive, et donc condamnable. Par exemple, les autres personnages la jugent négativement et s'éloignent d'elle, effrayés par sa prestance : «This person she'd become today had no kindness, no joy » (386). Sa violence devient la source d'une culpabilité insoutenable et fait l'objet d'une quête de rédemption qui lui restituera ultimement sa non-violence. Ce phénomène n’est pas nouveau selon 
Sharon Macdonald qui observe que la figure guerrière mythique et légendaire, « However, her intrinsic disruptiveness, as woman warrior, is effectively negated in the recasting of her story in ways which are, perhaps, symptomatic of the means by which female warrior figures, or strong women in general, are 'disarmed' » (7). Kristen Marthe Lentz montre quant à elle que dans de nombreux cas l'héroïne est victime d'une situation d'oppression dans laquelle elle n'a pas le choix d'utiliser autre chose que la violence afin de survivre. Lorsque Celaena prend délibérément les armes, elle le fait en réponse à une violence dont elle est victime. Lors de ses missions d'assassinats, elle n'a bien évidemment pas d'autres choix que de se battre afin de ne pas mourir. De plus, si elle refuse d'obéir aux ordres et de tuer les individus demandés, le roi lui promet une mort certaine.

Le morcellement des performances de genre chez la guerrière sert à négocier sa violence et à montrer une certaine complexité identitaire sans que rien ne soit affecté ou contaminé. Sur l'ensemble du récit, la guerrière peut être féminine et violente, mais pas de manière simultanée. L'usage de violence s'accomplira dans le cadre d'une performance masculine et donc dissociée de la féminité, ce qui explique pourquoi les personnages de guerrières revêtent fréquemment de nombreuses identités qu'elles empruntent selon les circonstances du récit. Comme nous le verrons, cette négociation de la violence peut prendre plusieurs formes se logeant tant dans le dispositif textuel qu'à travers la représentation visuelle, qui devient aussi discursive.

Le parcours initiatique guerrier de Celaena comporte une mise à l'écart de la féminité, laquelle ne disparait jamais complètement, mais reste circonscrite en lieu sûr. Elle cultive certes de nombreux traits considérés comme féminins, sauf que ceux-ci demeurent clairement dans l'ombre de ses activités principales. Par exemple, le personnage de Celaena voue un culte pour les beaux vêtements, la lingerie fine et le maquillage, passions qu'elle assouvit discrètement dans l'intimité de ses appartements. Cette délimitation identitaire donne naissance à deux versions de Celaena réparties de manière normative à travers le récit, soit la guerrière masculine lors des actions publiques, et la princesse féminine dans la sphère privée. La Celaena de la chambre à coucher se permet d'être sensible et de développer des amitiés profondes, de laisser tomber un instant son attitude arrogante pour se montrer vulnérable. En témoignent ces moments d'introspection où elle observe son reflet au miroir dans ses appartements : "Celaena stood before the rosewood mirror, smiling. She ran down a hand down her gown. Sea-foam white lace bloomed from the sweeping neckline, washing upon her breast from the powder-green ocean of 
silk that made up the dress » (61). Les soieries et la dentelle de la robe suggèrent la douceur féminine, mais aussi le caractère intime de cette rencontre secrète avec soi. D'un autre côté, comme nous l'avons, la guerrière apparaît sur la place publique littéralement et métaphoriquement impénétrable et indépendante : «Absolute confidence, absolute arrogance: her best shields and most beloved masks » (25) L'emploi du terme masque signale dès lors la dimension performative de l'attitude qu'elle arbore en société, qui se révèle aussi être son bouclier mental («shield $»)$.

\section{Guerrière en colère}

Celaena est engagée comme assassine par le roi et est obligée de tuer sous les ordres de ce dernier. Comme le souligne Frances Early dans Athenas's Daughters, la participation même d'une femme en tant que héros guerrier, comme c'est le cas de Celaena, est dangereuse du fait qu'elle place celle-ci au service du patriarcat et de l'agressivité (2). Cette observation éclaircit l'un des éléments constitutifs les plus problématiques de la femme violente, soit celui de la colère au féminin. En effet, comme le remarque Tania Modleski, la rage et la colère féminines sont toujours les émotions les plus inacceptables dans les registres des représentations de la femme (10). Ceci est d'autant plus significatif au sein de personnages féminins dont la fonction primaire est de se battre. Cet inconfort réside en la nature guerrière, car elle profane en son concept même la sacralité du féminin non violent, ce qui génère une dette à restaurer à travers sa représentation. Autrement dit, la représentation de sa violence doit être constamment justifiée dans les œuvres de culture populaire. Un exemple de cette légitimation est celui de Joss Whedon, le créateur de la série «Buffy la chasseuse de vampires », qui explique avoir mis un point d'honneur à ce que ses vampires arborent des visages difformes et volent en poussières en mourant. Il était pour lui inconcevable de représenter une jeune femme tuant des êtres humains (Neroni 65). Il est intéressant de lier cette obscénité de la violence avec celle de la colère au féminin qui secoue le tabou social de l'agressivité féminine (Douglas 32). Quant à elle, Hilary Neroni, s'est interrogée sur les manières dont la violence du héros féminin est négociée au cinéma. Elle pose le constat selon lequel un film peut neutraliser l'insupportable violence féminine en l'expliquant ou en la justifiant (25). On peut étendre cette théorie et proposer que tout texte, dans son dispositif, est potentiellement en mesure de contrebalancer la violence de ses personnages. 
Comme le remarque Neroni, les efforts déployés pour légitimer la femme violente témoignent du trauma causé initialement par son usage même de violence. La volonté d'atténuer la violence du personnage féminin repose sur une idéologie fondée sur le dualisme qui persiste à travers les normes de genre régissant l'imaginaire populaire et qui, comme le signale Rowe, identifie l'homme au sadisme et relie la femme au masochisme en raison de leur position respectivement active et passive. Or, dans la fiction, la violence féminine demeure sous la bannière de la protection de soi ou des autres, ainsi que l'avait observé Lisa Purse avec son trope de la «angry woman », celle qui ne tue que par altruisme ou en cas d'extrême nécessité (185198). Par exemple, dans Throne of Glass, le statut d'assassine de Celaena est, comme l'ont remarqué plusieurs lectrices, plutôt ironique dans le sens où la guerrière ne tue pratiquement aucun personnage de toute la série, et que ses rares victimes méritaient leur sort.

Une scène singulière, relatée du point de vue de son amant Chaol, la présente succombant à une rage meurtrière dans laquelle elle prend goût à la violence : «She was a whirlwind of steel and blood [...] he saw the lethal predator he'd expected to find in the mines. There was nothing human in her eyes, nothing remotely merciful. It froze his heart. [...] she was soaring through them, delighting in the feral song that sang through her blood and bones » (55).

Celaena éprouve du plaisir à tuer lorsqu'elle donne libre court à ses pulsions animales liées à ses lointaines origines féériques, quand elle s'abandonne à sa nature profonde, instinctive et agressive. L'animosité prédatrice de Celaena traumatise Chaol et cause la rupture du couple. Comme le souligne Rowe, les femmes agressives ne sont pas dangereuses en raison de leurs intentions, mais parce que leur colère dérange les normes de genre (42). L'aspect monstrueux de la colère et de la violence au féminin consiste en une déstabilisation idéologique. En ce qui concerne les guerrières, leur rage lègue son lot de remords et initie un examen de conscience chez les personnages. Les figures spectrales hantent aussi la conscience de Celaena et lui rappellent sa brutalité et les décès qu'elle a causés de près ou de loin : "She was living the same story again and again. The night she'd been captured, she'd also snapped, and come so close to killing the person she most wanted to destroy before someone knocked her out and she awoke in a rotting dungeon. She smiled bitterly as she opened her eyes. It was always the same story, the same loss » (345). Comme le signale ce passage, les multiples décès corrélatifs à ses combats font de la jeune femme un être tourmenté et constamment endeuillé. La guerrière de Throne of Glass souffre de traumatismes liés à la violence proférée. L'auteure en dévoile les conséquences 
psychologiques et morales : «I'll never forget the people I've killed [...] Even the ones I killed to survive. I still see their faces, still remember the exact blow it took to kill them. [...] No matter the cause, though, it - it still takes away a little piece of you each time. So I don't think I'll ever forget them » (24). C'est par la récurrence de figures revenantes, la réitération de pensées traumatiques et de phrases culpabilisantes (auxquelles nous avons accès grâce à la focalisation interne), que le récit souligne avec une volonté réaliste les blessures psychiques liées à la violence. Le corps de Celaena subit aussi des dommages physiques permanents, ainsi qu'en témoignent ses innombrables cicatrices, qui constituent autant d'inscriptions de son vécu. La vision de ses cicatrices provoque chez les personnages masculins de vives réactions : certains se montrent paternalistes, tel que son ex-maître assassin Arobynn Hamel : «It hurts my heart to see so many new scars on you » (17). Cependant, d'autres, comme son amoureux Rowan, éprouvent un sentiment empathique : «He'd seen the scars [...] peeking over the collar of her shirt every now and then. He hadn't yet dared to ask to see them. The bandaged bite on her arm was nothing compared to that pain and the many others she hadn't mentioned, the scars all over her » (218). Pour l'héroïne, la cicatrice représente le coût de la violence marquée sur sa chair, comme un témoin visible de sa conscience meurtrie. La culpabilité liée à la violence est le moteur d'une quête expiatrice qui restituera ultimement le caractère non violent de la jeune fille. Sitôt la violence proférée, le remords assaille Celaena et lui fait rechercher la rédemption, son parcours s'orientant désormais vers la maîtrise de sa colère. Dès lors, à travers l'objectif de reconstruction de son royaume déchu, elle apprend à canaliser et à rediriger sa nature enflammée dans des actions constructives d'ordre humanitaire. Par exemple, elle tendra la main à l'une de ses ennemies, Lady Kaltain, une prostituée désœuvrée, et fera d'elle son alliée en la formant au combat. Ultimement, Celaena se sacrifiera au nom de la communauté, offrant son existence aux dieux exigeant une rançon en échange de la libération de son peuple : «She would be the cost to end this » (622). « Suite à son autosacrifice, le lecteur apprend que Celaena planifiait son départ depuis longtemps, car elle avait prévu des directives pour chacun des membres de son entourage. On prend alors connaissance de l'ampleur de l'abnégation de la guerrière ».

Le concept de personnage antagoniste est issu du schéma actantiel classique selon Algirdas Julien Greimas, qui oppose de manière dichotomique le héros et son ennemi. Cependant, la ligne est mince entre la bonne et la mauvaise guerrière. Ce qui les distingue n'est souvent qu'une nuance dans l'aptitude à l'abnégation. Ainsi, la mauvaise guerrière a tendance à faire des choix 
égocentriques et vengeurs. L'héroïne guerrière positive, comme le souligne Kameron Hurley, doit se montrer responsable et pleine de vertu. Elle apparait encore plus vertueuse lorsqu'elle est justement mise en contraste avec une figure dérogeant à la féminité sacrificielle et refusant tout compromis. Dans Throne of Glass, le phénomène de repoussoir le plus significatif est celui entre Celaena et Ansel of Briarcliff. Ansel est l'héritière exilée d'un royaume dont elle cherche à regagner le pouvoir; dès lors se profile une similitude avec la quête de Celaena, qui est de sauver sa terre natale des mains ennemies. De plus, les deux jeunes femmes sont toutes aussi orgueilleuses, fières et têtues l'une que l'autre. Si elles deviennent rapidement amies, la trahison ne tardera pas à survenir lorsque Ansel tentera d'assassiner Celaena. Les reproches que Celaena adresse à Ansel pourraient aussi bien s'appliquer à elle-même : «After that, she'd sworn never to trust girls again, especially girls with agendas and power of their own » (101). Celaena peut à juste titre être considérée comme une héroïne ayant ses propres plans et qui use de tous les moyens possibles pour parvenir à ses fins. La principale différence entre leurs moralités réside dans le fait que les plans obscurs de Celaena visent le bien-être collectif alors que ceux d'Ansel sont tournés vers la restitution de son pouvoir individuel. La création de ce doppelgänger sanguinaire aurait pour effet de faire paraître Celaena plus raisonnable et responsable tout en atténuant sa violence, ce qui facilite sa réception auprès du public.

\section{Réception et polémique}

Une lecture de l'interprétation du public nous permet d'évaluer comment est compris le personnage. Rappelons ici John Fiske pour qui la signification d'un produit culturel est un procédé de cocréation qui ne se situe pas seulement du côté du texte, mais passe aussi par la lecture dont en fait le public (10). Cette analyse est d'autant plus importante dans le cas d'une figure imaginaire telle que la guerrière étant donné que « [1]a figure est une énigme qui ne reste pas statique, mais génère des interprétations $[\ldots] »$ (Gervais 17). Notre recherche veut donc tenir compte de l'interprétation du public afin d'évaluer comment on comprend et on s'approprie l'héroïne de Maas.

À travers nos visites chez les communautés virtuelles de fans, nous remarquons que les lectrices $^{2}$ de Throne of Glass tendent à s'approprier le personnage et à le remettre en scène à travers des transfigurations qui témoignent de son morcellement identitaire. Si Maas met en circulation deux rôles et représentations visuelles pour son personnage, les créations des fans 
dépeignent souvent ces deux «personnifications » au sein d'une seule et même image. Ainsi, les illustrations des fans, qui présentent côte à côte deux figurations de Celaena, l'une en robe, l'autre en tenue de combat, opèrent une séparation genrée similaire à celle du texte.

Dans son dispositif textuel, l'héroïne de Maas installe d'emblée une double lecture à l'égard de la problématique de genre. D'une part, elle reconduit un schéma binaire divisant de manière nette le féminin et le masculin. D'autre part, le jeu de rôle genré qu'elle inspire est repris par les fans qui explorent les identités sexuées. À ce sujet, notre opinion va dans le sens de celle de Rosi Braidotti, pour qui on peut choisir de revisiter la stéréotypie de manière labile et de travailler à partir de celle-ci, au lieu de la rejeter. Selon elle, il est possible de jouer avec les frontières des genres en y retournant consciemment. Dans cet esprit, c'est parce qu'elle met en évidence le côté reconstructible des rôles genrés que Celaena incite l'exploration des performances de genre.

Le terme performance prend ici tout son sens, car le lectorat choisit de s'approprier ce qui lui est d'emblée présenté comme étant des rôles dans l'architecture de l'œuvre. Poststructuralisme et postmodernisme contemporains alimentent l'idée d'une conceptualisation identitaire fragmentée, fluide et mouvante, de l'ordre de la performance. D'ailleurs, Rowe expose le fait qu'en accord avec les principes post-structuralistes et ses notions de construction de sujets et d'identités fluides, les publics contemporains conçoivent davantage les catégories identitaires comme des marqueurs mobiles qui peuvent être empruntés, performés et renversés de manière ironique, ludique, ou encore avec une intention politique.

Concernant les critiques qu'elle génère, Celaena est tantôt dénigrée, tantôt adorée. Si l'on se fie aux contrastes entre les discours des fans par rapport à sa construction identitaire, la guerrière de Maas soulève clairement un débat sur la violence au féminin. À titre d'échantillon, le débat idéologique au sein des commentaires des lectrices sur la plateforme littéraire Goodreads est éloquent. Du côté des critiques négatives, la plainte la plus répandue est que Celaena est trop féminine pour incarner une assassine crédible. En voici un exemple assez explicite : «The characterization was inconsistent to me - she's supposed to be the most feared assassin in the land, and she does spend time thinking about how easy it would be to kill her 'captors', yet at times she acted like a young girl » (M.S. 12 avril 2014). Il est vrai que l'auteure dépeint son hérö̈ne de manière à présenter tantôt son côté impitoyable et violent, tantôt son côté sensible et vulnérable, tout en évitant de les mélanger. En quoi le fait d'agir comme une jeune 
fille est-il incompatible avec le meurtre ? Ce commentaire perpétue la vision considérant la féminité et l'agressivité comme deux entités hétérogènes. Dans la même veine, certaines lectrices perçoivent cette combinaison de féminité et de violence comme une incohérence et en sont par conséquent fortement contrariées : «Amidst all these competitions and training programs, the plot scattered into flirting banter, gatecrashing parties, and some girl-on-girl hating ; as if the author wasn't quite sure whether she wanted her novel to be the story of a ruthless assassin, or the romances of a princess-wannabe » (L.M., 12 août 2012). On comprend ici que le récit d'une jeune femme ne peut se constituer de combats et de bal masqués sans courir le risque de perdre toute crédibilité. Curieusement, Celaeana reçoit le mépris et l'admiration pour des raisons pratiquement identiques : «I did appreciate her for she was simultaneously flirtatious and feminine while maintaining her aura of kick-ass and deadly » (K., 20 août 2012). L'une de ses adhérentes s'élève même contre les critiques à l'endroit du côté féminin du personnage : «I've read several reviews for this book and the most common complaint is that Celaena is too much of a girly-girl to be a believable assassin. To that I say, nonsense. (...) In fact, a love of clothes and a preoccupation with appearance is consistent with being an assassin, in my opinion » (R.R., 12 novembre 2015). Ainsi, certaines lectrices défendent la combinaison de féminité et de violence comme une identité légitime Elles envisagent une possibilité là où d'autres ne lisent qu'une maladresse d'écriture, et en cela s'inscrivent dans une conception poststructuraliste de l'identité comme construction labile et non fixe. Cet accueil favorable encourage une telle diversité chez les nouveaux personnages de sexe féminin. La divergence des opinions à l'égard de Celaena témoigne du fait que la féminité agressive est toujours loin d'être unanimement acceptée auprès des lectorats.

\section{Conclusion}

Compte tenu du fait que le désir initial de l'auteure était de créer une héroïne affranchie des normes, force est de constater que Celaena, sur les plans identitaire et représentatif, ne demeure que partiellement marginale. Si elle s'aventure vers une forme de transgression en proposant une identité articulant violence et féminité, cette combinaison demeure préventivement fragmentée et négociée. Malgré sa fragilité, l'esquisse liminale qu'elle propose reçoit une lecture nettement polémique de la part du public, débat qui ne fait que confirmer la persistance d'une dissonance entre féminité et violence dans nos imaginaires collectifs. 
Rejoignant cette idée, on peut expliquer la réaction partagée du lectorat face à la marginalité suggérée par le personnage de Celaena avec Rowe, selon qui le pouvoir associé au sujet divergent est ambivalent et serait souvent retourné contre ce dernier (41). Même si l'héroïne de Throne of Glass n'est peut-être pas aussi ingouvernable qu'elle ne le laisse entendre, nous ne pouvons nier le pouvoir subversif qu'elle contribue à installer au sein de la littérature populaire, car comme le souligne Rowe, « la liminalité est aussi une source de pouvoir dans laquelle prend sa source la femme ingouvernable : le pouvoir de déstabiliser les cadres archaïques pour en créer de nouveaux » (traduction libre 41). La réponse du public prend de plus en plus d'espace, de visibilité et de légitimité dans la sphère publique ; elle migre, donc, des marges vers le centre de production. En raison de cette convergence entre l'œuvre et sa réception, la culture fanique pourrait éventuellement devenir une source influente de renouvellement de la figure guerrière auprès des auteurs et permettrait d'assouplir la tension entre violence et féminité.

\section{Bibliographie}

Agamben, Giorgio. Profanations. Paris : Rivages, 2006.

Braidotti, Rosi. «On the Feminist Female Subject or from She-self to She-other. In G. Bock and S. James. Beyond Equality and Difference : Citizenship, Feminist Politics and Female Subjectivity. Londres : Routledge, 1992. 92-176.

Cixous, Hélène. Le rire de la Méduse et autres ironies. Paris : Galilée, 2010.

Collins, Suzanne. The Hunger Games Trilogy : Hunger Games. New York : Scholastic P, 2008.

---. The Hunger Games Trilogy : Catching Fire. New York : Scholastic P, 2009.

---. The Hunger Games Trilogy : Mockingjay. New York : Scholastic P, 2010.

Douglas, Mary. Purity and Danger. New York : Routledge, 2003.

Dufourmantelle, Anne. La femme et le sacrifice. D'Antigone à la femme d'à côté. Paris : Denoël, 2007.

Early, Frances et Kathleen Kennedy. Athena's Daughters : Television's New Women Warriors. New York : Syracuse UP, 2003.

Fiske, John. Understanding Popular Culture. Boston: Unwin Hyman, 1989.

Fuss, Diana. Essentially Speaking : Feminism, Nature and Difference. Londres : Routledge. 
Gallucci, Kelly. « Sarah J. Maas on the Inspiration for Queen of Shadows That She's Kept Secret for 14 Years ». Bookish, 2 septembre 2015. https://www.bookish.com/articles/sarah-jmaas-on-the-inspiration-for-queen-of-shadows-that-shes-kept-secret-for-14-years/

Gervais, Bertrand. Figures, lectures. Logique de l'imaginaire I. Montréal : Le Quartanier, 2007.

Goodreads. The Assassin's Blade: Throne of Glass Novellas. https://www.goodreads.com/book/show/18243700-the-assassin-sblade?from_new_nav=true $\& a c=1$

---. Throne of Glass (Throne of Glass 1). https://www.goodreads.com/book/show/7896527throne-of-glass?from_new_nav=true \&ac=1

---. Crown of Midnight (Throne of Glass 2). https://www.goodreads.com/book/show/17167166crown-of-midnight?from new nav $=$ true $\&$ ac $=1$

---. Heir of Fire (Throne of Glass 3). https://www.goodreads.com/book/show/20613470-heir-offire?from_new nav $=$ true $\& a c=1$

---. Queen of Shadows (Throne of Glass 4). https://www.goodreads.com/book/show/18006496queen-of-shadows?from_new nav $=$ true $\& a c=1$

---. Sarah J. Maas. https://www.goodreads.com/author/show/3433047.Sarah_J_Maas

Greimas, A. J. Sémantique structurale. Paris : P.U.F. (1986) [1966].

Inness, Sherrie. Tough Girls : Women Warriors and Wonder Women in Popular Culture. New York : Palgrave Macmillan, 1999.

Rowe, Kathleen Karlyn. Unruly Girls, Unrepentant Mothers. Redefining Feminism on Screen. Austin : U of Dallas P, 2011.

---. The Unruly Woman : Gender, and the Genres of Laughter. Austin : U of Texas P, 1995.

Mass, Sarah J. Throne of Glass. New York : Bloomsbury P, 2012.

---. Crown of Midnight. New York : Bloomsbury P, 2013.

---. Heir of Fire. New York : Bloomsbury P, 2014.

---. The Assassin's Blade. The Throne of Glass Novellas. New York : Bloomsbury P, 2014.

---. Queen of Shadows. New York : Bloomsbury P, 2015.

---. Empire of Storms. New York : Bloomsbury P, 2016.

Modleski, Tania. Feminism Without Women : Culture and Criticism in a 'Postfeminist' Age. New York : Routledge, 1991. 
Neroni, Hilary. The Violent Woman. Femininity, Narrative, and Violence in Contemporary American Cinema. New York : State U of New York P, 2005.

Notes

${ }^{1}$ Selon Henry Jenkins, l'un des critères de base de la culture populaire est l'ampleur de la réception des œuvres. (2) Best-seller, la série Throne of Glass est massivement lue à travers le monde.

${ }^{2}$ Nos observations sur les forums et réseaux sociaux nous confirment que la quasi-totalité du lectorat visible de Throne of Glass est de sexe féminin. L'existence de lecteurs masculins n'est certes pas exclue, mais ceux-ci ne semblent pas se manifester publiquement. 